\title{
Varied Presentations of Absent Ductus Venosus, Their Associations, and Outcomes
}

\author{
Anuja Thomas $^{1}$ (D) Bijoy K. Balakrishnan ${ }^{1} \cdot$ Meenu Batra $^{1} \cdot$ Rekha Raichel Kuriakose $^{1} \cdot$ \\ Seneesh Kumar Vikraman ${ }^{1} \cdot$ Kannoly Karunakaran Gopinathan ${ }^{1}$
}

Received: 3 August 2015/Accepted: 13 November 2015/Published online: 9 December 2015

(C) Society of Fetal Medicine 2015

\begin{abstract}
The present study aimed at identification of fetuses with absent ductus venosus at prenatal screening ultrasound, determination of the type of umbilical venous drainage, and follow-up to assess their outcomes. This prospective study was conducted at CIMAR Hospital, Kerala, in antenatal women with singleton pregnancy, who underwent screening at 11 to $13+6$ weeks, from November 2013 to January 2015. Ultrasonography was done according to Fetal Medicine Foundation (FMF) protocol. Absence of ductus venosus was diagnosed, when ductus venosus was not visualized connecting the portal sinus to the subphrenic confluence. The type of umbilical venous drainage was determined. Karyotyping was offered in all diagnosed cases and follow-up was obtained. Absent ductus venosus was diagnosed in three out of the 3691 women screened. Two of these had intrahepatic and the third had extrahepatic type of umbilical venous drainage. Karyotype was normal in all the three. The first patient with intrahepatic type of drainage developed polyhydramnios and ended up in intrauterine fetal demise at 26 weeks of gestation. In the second patient, malaligned ventricular septal defect and intestinal obstruction was detected in the anomaly scan. She underwent termination of pregnancy. The fetus with extrahepatic type of umbilical venous drainage developed cardiomegaly on follow-up. This baby underwent ligation of patent ductus arteriosus at third month of life. Our study suggests that absence of ductus venosus is associated with variable fetal outcome. Careful
\end{abstract}

Anuja Thomas

dranujathomaz@gmail.com

1 Department of Feto Maternal Medicine, CIMAR Hospital, Off NH-17, Thykkavu Bus Stop Cheranaloor, Edappally, Kochi, Kerala 682034, India search of co-existing anomalies and follow-up is warranted in these cases.

Keywords Absent ductus venosus - Umbilical venous drainage · Cardiomegaly · Polyhydramnios · Intrauterine death

\section{Introduction}

Ductus venosus is a short vascular channel in the fetal abdomen connecting the intra-abdominal portion of the umbilical vein to the left side of the inferior vena cava. (Fig. 1) It is $5-17 \mathrm{~mm}$ long and trumpet shaped, the isthmus measurement increases from $0.5 \mathrm{~mm}$ at mid-gestation to $2 \mathrm{~mm}$ during the rest of the gestation and outlet width from 1.25 to $3 \mathrm{~mm}$ [1-4]. The reduction of diameter produces a pressure gradient between the umbilical vein and the atrium resulting in an acceleration in blood flow in the ductus $[1,2]$. This velocity increases with gestational age [1]. It is estimated that $20 \%-30 \%$ of the oxygenated blood of the umbilical vein enters the ductus venosus and directly reaches the left heart across the foramen ovale [4]. Ductus venosus plays a major role in the regulation of fetal circulation by modifying the volume of its flow depending on the pressure gradient between the umbilical vein and the heart.

The DV waveform has three phases [1] (Fig. 2):

S highest velocity peak, corresponding to ventricular systole

D next peak reflecting early diastole

a lowest velocity forward flow corresponding to atrial contraction 


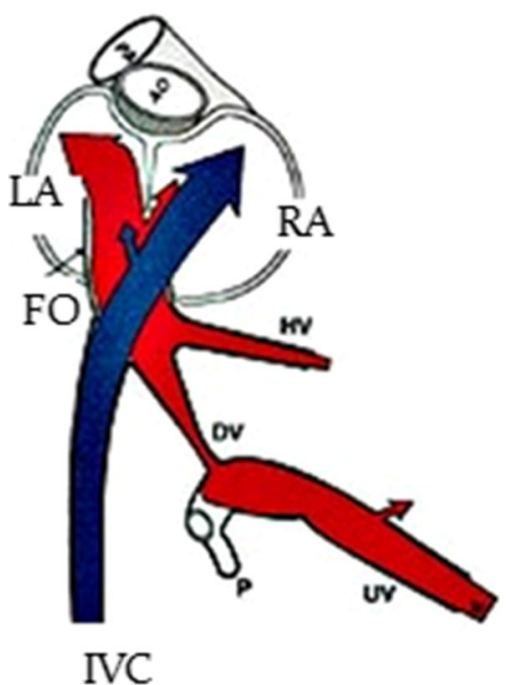

Fig. 1 Oxygenated blood from the umbilical vein (red color) directed through the ductus venosus (DV) and the left hepatic vein preferentially enters the left atrium (LA), while the deoxygenated blood (blue color) from the abdominal portion of the inferior vena cava enters the right atrium (RA) [5]

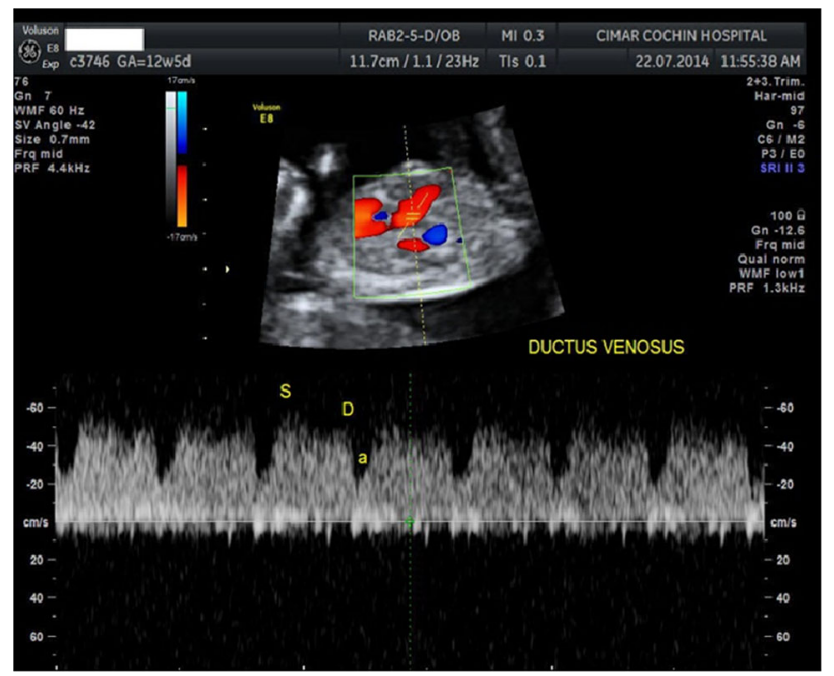

Fig. 2 Doppler assessment of ductus venosus showing the typical triphasic waveform

First trimester assessment of blood flow pattern across the ductus venosus is useful in screening for chromosomal abnormalities and major cardiac defects in the fetus [6,7]. Absence of ductus venosus is a rare but well-described abnormality (Fig. 3). The incidence of absent ductus venosus in general population is unknown. It has been reported by Staboulidou et al. [8] that the incidence of absent ductus venosus was one in 2532, in a population presenting for the 11 to $13+6$ weeks scan. Previously, studies have been conducted to assess the pregnancy outcome in absent ductus venosus diagnosed in second and

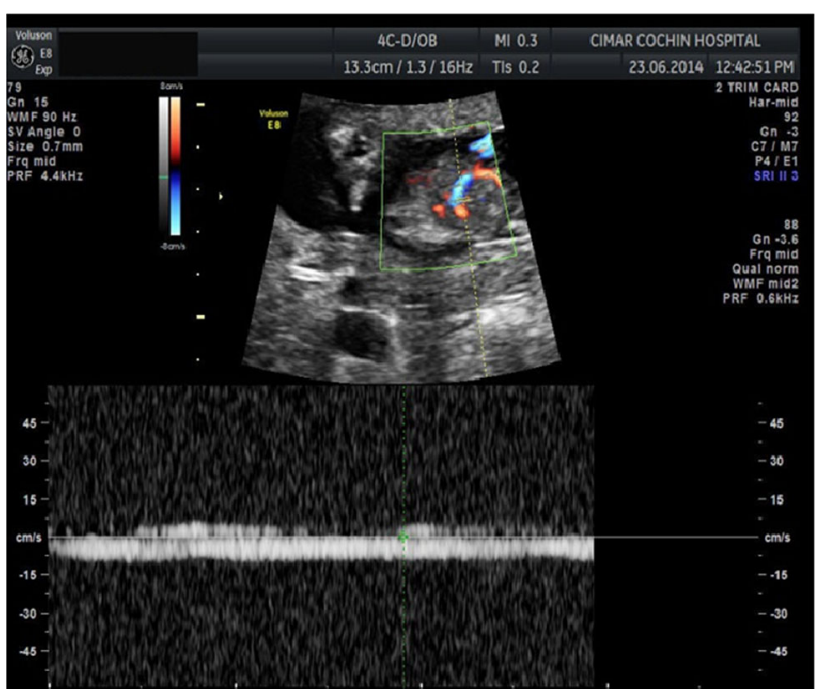

Fig. 3 Ultrasound image showing a case of absent ductus venosus

third trimester [9-14]. Analysis of previously reported cases of agenesis of ductus venosus shows that about $40 \%$ had associated defects and aneuploidies, and in pregnancies with isolated absent ductus venosus, about $35 \%$ resulted in termination or perinatal death [8].

Although many different varieties of abnormal venous circulation were documented, they can be mainly classified into extrahepatic and intrahepatic. In a retrospective study published in 2001, Contratti et al. [11] has mentioned three main patterns:

1. Extrahepatic variety with umbilical vein draining into the inferior vena cava by either one iliac or renal vein.

2. Extrahepatic variety with umbilical vein draining directly to the right atrium.

3. Intrahepatic variety with umbilical vein connecting to the portal circulation without giving rise to ductus venosus.

\section{Materials and Methods}

This was a prospective study conducted at CIMAR Hospital, Kerala from November 2013 to January 2015. All antenatal women with singleton pregnancy who underwent screening at 11 to $13+6$ weeks gestation, crown rump length (CRL) within $45-84 \mathrm{~mm}$, were included in the study. Multiple pregnancies were excluded. History was noted and clinical examination carried out and recorded. Ultrasound scan was performed transabdominally using 3-7.5 MHz curvilinear transducers on GE Voluson E8. Ultrasound was done according to Fetal Medicine Foundation (FMF) protocol and scans were done by sonographers who had obtained FMF certificate of competence in 
the 11-13 weeks scan. The examination was undertaken during a period of fetal quiescence. The image was magnified such that the fetal thorax and abdomen occupied the whole screen. A right ventral mid-sagittal view of the fetal trunk was obtained and color flow mapping done to demonstrate the umbilical vein, ductus venosus, and fetal heart. Ductus venosus is identified as a yellowish aliasing area just above the umbilical sinus [15]. The pulsed Doppler sample was kept small $(0.5-1.0 \mathrm{~mm})$ and placed in the area of aliasing. The angle of insonation was maintained below $30^{\circ}$ and filter frequency set low at $50-70 \mathrm{~Hz}$. The sweep speed was high, around $2-3 \mathrm{~cm} / \mathrm{s}$.

If ductus venosus was not visualized connecting the portal sinus to the sub-phrenic confluence, absent ductus venosus was diagnosed. The type of umbilical venous drainage was also determined. These women were counseled, karyotyping was offered in all cases diagnosed, and they were followed up with monthly scans, including a detailed anomaly scan and fetal echocardiography.

\section{Results}

During the study period, 3691 women underwent screening. Out of them, three women were diagnosed to have absent ductus venosus (Table 1). One of them had extrahepatic type of umbilical venous drainage with the umbilical vein draining directly into the right atrium. The other two patients had an intrahepatic type of umbilical venous drainage. Karyotyping was done in all the three patients, all of them turned to be normal.

The patient who had a direct drainage of the umbilical vein into the right atrium developed cardiomegaly in the subsequent follow-up at 24 weeks of gestation. She delivered a term female baby of $2.5 \mathrm{~kg}$ birth weight. Echocardiography was done postnatally which was normal. This child underwent ligation of the patent ductus arteriosus at third month of life, and is doing well now.

The second patient in our study, who had an intrahepatic type of umbilical venous drainage, was diagnosed to have associated cardiac anomaly-malaligned ventricular septal defect and intestinal obstruction, in her follow-up scan. This patient underwent termination of pregnancy elsewhere.
The third patient who also had an intrahepatic type of umbilical venous drainage was followed up. She did not have any associated structural anomalies. She developed polyhydramnios at 24 weeks of gestation and had intrauterine fetal demise at 26 weeks of gestation. The fetus looked morphologically normal. The result of autopsy is awaited in this case.

\section{Discussion}

We were able to identify three positive cases of absent ductus venosus in our study. The incidence in the population studied comes to one in 1230. This is higher than that mentioned in a study by Staboulidou et al. [8] in a similar population. Considering the types of umbilical venous drainage, two types were identified, the intrahepatic type in two cases and in one, extrahepatic with direct drainage of umbilical vein into the right atrium. This is similar to the findings of Berg et al. [13] who suggested that intrahepatic drainage of umbilical vein is more frequent than the extrahepatic type of absent ductus venosus.

We did not identify any chromosomal abnormalities with any of our cases. This was in contrast to findings of Staboulidou et al. [8] who identified a high prevalence of anueploidies in fetuses with absent ductus venosus.

Similar to the finding in the study by Berg et al. [13] who detected a significant association of cardiomegaly in fetuses with extrahepatic bypass, we identified cardiomegaly in a case of absent ductus venosus with umbilical vein draining directly into the right atrium. Persistence of patent ductus arteriosus in this case may be probably attributed to the cardiac overload and persistent pulmonary hypertension.

The second patient who had an intrahepatic type of absent ductus venosus had an associated cardiac anomaly and gastrointestinal malformation. Cardiac anomalies were the most common structural anomaly associated with absent ductus venosus [12, 13, 16-20].

Hyperdynamic circulation in absence of ductus venosus explains the mechanism behind the polyhydramnios developed in the third patient who had intrahepatic type of umbilical venous drainage. This has been documented in previous studies $[11,16,17,21,22]$.
Table 1 Ultrasound findings, fetal karyotype, and pregnancy outcome in the cases diagnosed with absent ductus venosus

\begin{tabular}{lllll}
\hline & Case & Type & Aneuploidy & Sonographic findings \\
\hline 1 & Outcome & & & \\
2 & Extrahepatic & Nil & Cardiomegaly & Normal delivery \\
3 & Intrahepatic & Nil & Cardiac, GIT malformations & Termination of pregnancy \\
\hline
\end{tabular}

GIT gastrointestinal tract 
Regarding the overall prognosis, the intrahepatic variety, which had associations like polyhydramnios, cardiac abnormality, and gastrointestinal malformation, had bad outcome in terms of intrauterine death and termination of pregnancy. The extrahepatic variety which was associated with cardiomegaly had a good outcome. Berg et al. [13] emphasized the importance of the type of umbilical venous drainage in predicting the outcome, where as Thomas et al. [23] did not support this. The latter concluded that the main determinant in the outcome in fetuses with absent ductus venosus was their associations with structural or chromosomal abnormality and functional cardiac overload. The study suggested that the fetuses with isolated agenesis of ductus venosus had a fairly good outcome. In the present study, it was difficult to draw a conclusion in this respect as we had limited cases only.

\section{Conclusion}

The present study suggests that absence of ductus venosus is associated with variable outcome. Determination of the type of umbilical venous drainage may help in prognostication, considering the impacts on fetal hemodynamics in each type. Absence of ductus venosus warrants careful search of associated chromosomal and structural anomalies and these fetuses need serial follow-up examinations.

\section{Compliance with Ethical Standards}

Conflict of interest None.

\section{References}

1. Kiserud T, Eik-NES SH, Blass HG, Hellevik LR. Ultrasonographic velocimetry of the fetal ductus venosus. Lancet. 1991;338:1412-4.

2. Kiserud T, Hellevik LR, Eik-NES SH, Angelsen BAJ, Blass HG. Estimation of the pressure gradient across the fetal ductus venosus based on Doppler velocimetry. Ultrasound Med Biol. 1994;20:225-32.

3. Kiserud T, Rasmunssen S. How repeat measurements affect the mean diameter of the umbilical vein and the ductgus venosus. Ultrasound Obstet Gynecol. 1998;11:419-25.

4. Kiserud T, Rasmunssen S, Skulstad S. Blood flow and degree of shunting through the ductus venosus in human fetus. Am J Obstet Gynecol. 2000;182:147-53.

5. Kiserud T, Rasmussen S. Ultrasound assessment of fetal foramen ovale. Ultrasound Obstet Gynecol. 2001;17:119-24.

6. Miaz N, Valenica C, Kagan KO, Wright D, Nicolaides KH. Ductus venosus Doppler in screening for trisomies 21, 18 and 133 and Turner syndrome at 11-13 weeks of gestation. Ultrasound Obstet Gynecol. 2009;33:512-7.
7. Cheleman T, Syngelaki A, Miaz N, Allan L, Nicolaides KH. Contribution of Ductus venosus Doppler infirst trimester screening for major cardiac defects. Fetal Diagn Ther. 2011;29:127-34.

8. Staboulidou I, Pereira S, Cruz JJ, Syngelaki A, Nicolaides KH. Prevalence and outcome of absent ductus venosus at $11\left(^{+0}\right)$ to 13 $\left({ }^{+6}\right)$ weeks. Fetal Diagn Ther. 2011;30:35-40.

9. Gembruch U, Baschat AA, Caliebe A, Gortner L. Prenatal diagnosis of ductus venosus agenesis: a report of two cases and review of literature. Ultrasound Obstet Gynecol. 1998;11:185-9.

10. Achiron R, Hegesh J, Yagel S, Lipitz S, Cohen SB, Rotstein Z. Abnormalities of the fetal central veins and umbilicoportal system:prenatal ultrasonographic diagnosis and proposed classification. Ultrasound Obstet Gynecol. 2000;16:539-48.

11. Contratti G, Banzi C, Ghi T, Perolo A, Pilu G, Visentin A. Absence of ductus venosus: report of 10 new cases and review of the literature. Ultrasound Obstet Gynecol. 2001;18:605-9.

12. Volpe P, Marasini M, Caruso G, Lituania M, Marzullo A, Volpe $\mathrm{G}$, et al. Prenatal diagnosis of ductus venosus agenesis and its association with cytogenetic/congenital anomalies. Prenat Diagn. 2002;22:995-1000.

13. Berg C, Kamil D, Geipel A, Kohl T, Hnsmann M, Gembruch U. Absence of ductus venosus-importance of umbilical venous drainage site. Ultrasound Obstet Gynecol. 2006;28:275-81.

14. Achiron R, Gindes L, Kivilevitch Z, Kuint J, Kidron D, Boyanover $\mathrm{Y}$, et al. Prenatal diagnosis of congenital agenesis of the fetal portal venous system. Ultrasound Obstet Gynecol. 2009;34:643-52.

15. Miaz N, Kagan KO, Milovanovic Z, Celik E, Nicolaides KH. Learning curve for Doppler assessment of ductus venosus flow at $11+0-13+6$ weeks' gestation. Ultrasound Obstet Gynecol. 2008;31:503-6.

16. Sau A, Sharland G, Simpson J. Agenesis of ductus venosus associated with direct umbilical venous return into the heart; case series and review of literature. Prenat Diagn. 2004;24:418-23.

17. Jaeggi ET, Fouron JC, Hornberger LK, Proul F, Oberhansli I, et al. Agenesis of the ductus venosus that is associated with extrahepatic umbilical ven drainage: prenatal features and clinical outcome. Am J Obstet Gynecol. 2002;187:1031-7.

18. Hofstaetter C, Plath H, Hansmann M. Prenatal diagnosis of the abnormalities of the fetal venous system. Ultrasound Obstet Gynecol. 2000;15:231-41.

19. Langman $\mathrm{G}$, Wainwright $\mathrm{H}$, Matthews L. Absence of the ductus venosus with direct connection between the umbilical vein and right atrium. Pediatr Dev Pathol. 2001;4:298-303.

20. Hille H, Chaoui R, Renz S, Hecher K. Distended azygouus and hemiazygous vein without interrupted vena cava in a case of agenesis of the ductus venosus. Ultrasound Obstet Gynecol. 2008:31:588-91.

21. Hoppen T, Hofstaetter C, Plath H, Kau N, Bartmann P. Agenesis of ductus venosus and its correlation to hydrops fetalis. J Perinat Med. 2000;28:69-73.

22. Jorgensen $\mathrm{C}$, Andolf $\mathrm{E}$. Four cases of absent ductus venosus: three in combination with severe hydrops fetalis. Fetal Diagn Ther. 1994;9:395-7.

23. Thomas JT, Petersen S, Cincotta R, Lee-Tannock A, Gardener G. Absent ductus venosus-outcomes and implications from a tertiary centre. Prenat Diagn. 2012;32:689-91. 